\title{
Biofilm Structure of Geobacter sulfurreducens by Helium Ion Microscopy
}

\author{
A. Belianinov, ${ }^{1,2,3}$ M. C. Halsted, ${ }^{1,3}$ M. J. Burch, ${ }^{1,2}$ K. Songkil, ${ }^{1,2}$ and S. T. Retterer ${ }^{1,2,3,4}$ \\ 1. Center for Nanophase Materials Sciences, Oak Ridge National Laboratory, Oak Ridge TN \\ 2. The Institute for Functional Imaging of Materials, Oak Ridge National Laboratory, Oak Ridge, TN \\ 3. The Bredesen Center, The University of Tennessee, Knoxville, TN \\ 4. Biosciences Division, Oak Ridge National Laboratory, Oak Ridge, TN
}

Microbial communities form biofilms on material surfaces in a multitude of ecosystems, from the root hairs of a plant to the human gut. The hallmarks of an established biofilm include (1) the attachment of microbial cells to a surface, (2) production of extracellular polymeric substance, (EPS) (3) a complex structure or "architecture," and (4) the ability to exchange genetic information between cells. [1] Geobacter sulfurreducens forms unique, electrically conductive biofilms, a property that can be exploited in production and design of microbial fuel cells. In this work, examine biofilm formation, and biofilm properties of Geobacter sulfurreducens using a Scanning Electron Microscope (SEM) as well as a Helium Ion Microscope (HIM).

SEM is a high-resolution imaging technique used for characterization of a broad variety of materials. However, in order to image highly insulating, soft biological materials, the samples must be coated for charge compensation. These (typically) metallic coatings create a homogenous surface and may cloak true biological behavior and material contrast in the micrograph. In the case of Geobacter sulfurreducens, metal coating precludes detailed investigation of microbial attachment, presence of EPS, and fine surface details that may elucidate the mechanisms behind architecture formation and genetic material exchange.

Recently introduced HIM, offers more flexibility in investigating biological samples, as highly insulating sample can be imaged sui generis, without the use of a conductive coating. [2] This opens new pathways to capturing high resolution spatial details of biofilm formation and biofilm properties. Furthermore, high-resolution HIM imaging reveals true surface details of Geobacter sulfurreducens, such as flagella or pilin typically inaccessible by SEM. Finally, the effects of different sample preparation strategies for SEM and HIM will be illustrated and discussed [3].

\section{References:}

[1] Donlan, R. M. Emerging Infectious Diseases, 8(9) (2002), p. 881.

[2] Joens, M. S. et al, Scientific Reports 3 (2013), p. 3514.

[3] This research was supported by Oak Ridge National Laboratory's Center for Nanophase Materials Sciences (CNMS), which is sponsored by the Scientific User Facilities Division, Office of Basic Energy Sciences, U.S. Department of Energy. 


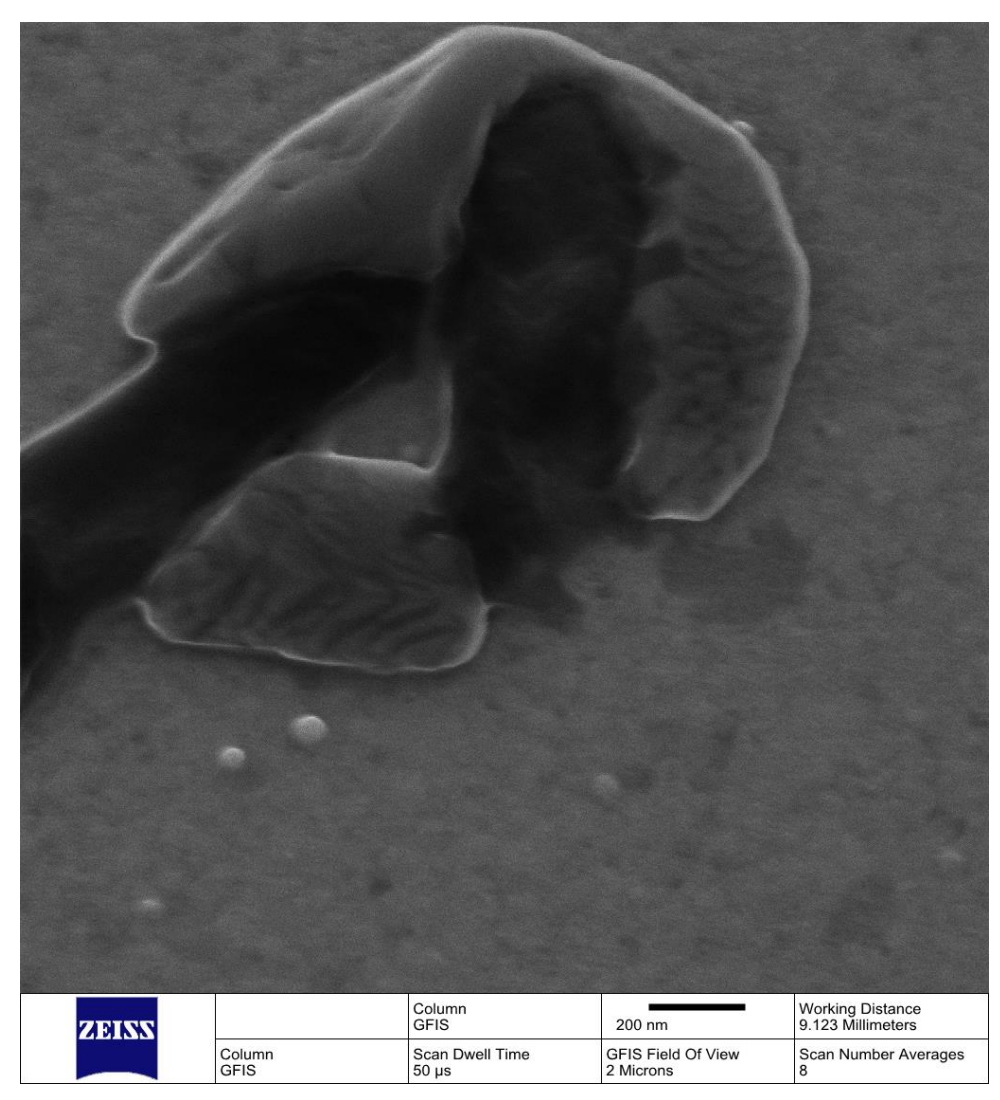

Figure 1. Helium Electron Microscope micrograph of Geobacter sulfurreducens. Sample preparation: 1 $\mathrm{mL}$ of $4 \%$ formaldehyde was pipetted onto glass coverslip with Geobacter sulfurreducens attached, and left for 5 minutes. Sample was tilted, washed twice with $0.5 \mathrm{~mL}$ of phosphate buffer saline solution, and then rinsed with $95 \%$ ethanol.

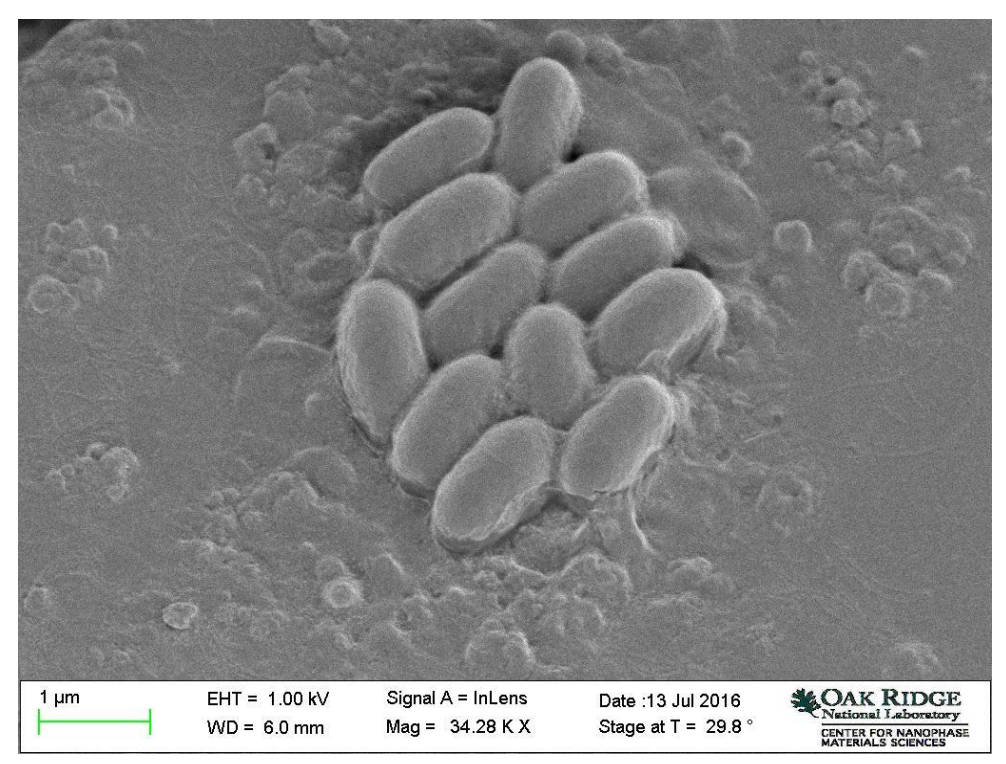

Figure 2. Scanning Electron Microscope micrograph Geobacter sulfurreducens, with a $10 \mathrm{~nm}$ of chromium (no chemical of physical fixation) coating prior to imaging. 